\title{
Prolonged Survival with Regorafenib in Metastatic Cholangiocarcinoma: Case Report
}

\author{
Costa CM, Gongora ABL, Lopes CDH, Salgues ACR and Capareli FC* \\ Oncology Center, Hospital Sírio-Libanês (HSL), Brazil
}

ISSN: 2639-0531

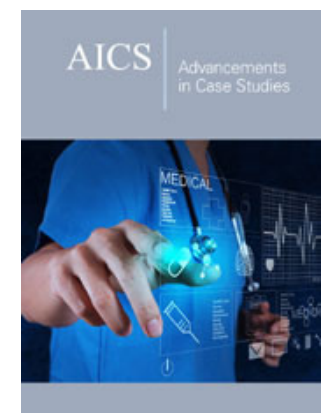

*Corresponding author: Capareli FC, Oncology Center, Hospital Sírio Libanês, Brazil

Submission: 归 March 14, 2020

Published: 制 April 14, 2020

Volume 2 - Issue 4

How to cite this article: Costa C, Gongora A, Lopes C, Salgues A, and Capareli F. Prolonged Survival with Regorafenib in Metastatic Cholangiocarcinoma: Case Report. Adv Case Stud.2(4). AICS.000542.2020

DOI: 10.31031/AICS.2020.02.000542

Copyright@ Capareli FC, This article is distributed under the terms of the Creative Commons Attribution 4.0 International License, which permits unrestricted use and redistribution provided that the original author and source are credited.

\begin{abstract}
Background: Advanced cholangiocarcinoma is a rare, aggressive tumor. Treatment is limited and very few systemic therapies are available. An effort for new drugs development is in course, which aims higher response rates, prolonged survival and better quality of life. Currently, immune checkpoint inhibitors and tyrosine kinase inhibitors have been used as new approaches after first- or second-line chemotherapy.

Case Presentation: A 49-year-old woman, diagnosed in 2013 with an intrahepatic cholangiocarcinoma, underwent surgery (pT2pN1) followed by adjuvant capecitabine and radiotherapy. Disease relapse occurred 9 months later, with a unique hepatic lesion that was treated with gemcitabine and cisplatin following liver ablation by radiofrequency. After 2 years, the identification of metastatic lesions in liver, lymph nodes and bones lead the patient to a re-exposure to gemcitabine and cisplatin, which resulted in partial tumor response, accompanied by limiting myelotoxicity induced by chemotherapy. A combination of fluorouracil, leucovorin and irinotecan was started, but disease progressed after 7 cycles. The patient received regorafenib in a third line setting, which resulted in a substantial metabolic response in all secondary lesions. The patient remains with stable disease for more than 16 months.
\end{abstract}

Conclusion: A surprising prolonged survival with regorafenib was observed. Selecting molecular targets is a promising field in the precision oncology.

Keywords: Advanced cholangiocarcinoma; Regorafenib; Vascular endothelial growth factor inhibitor; Gastrointestinal oncology; Case report

Abbreviations: CG: cisplatin and gemcitabine; CT: chemotherapy; ECOG: Eastern Cooperative Oncology Group; FDG: fluorodeoxyglucose; FGFR1: fibroblast growth factor receptor 1; mFOLFOX: modified fluorouracil, leucovorin and oxaliplatin; GI: gastrointestinal; ICC: intra-hepatic cholangiocarcinoma; IDH: isocitrate dehydrogenase; mIDH1: mutant IDH1; dMMR: DNA mismatch repair deficiency; MSI-H: high microsatellite instability; NGS: next generation sequencing; NTRK: neurotrophic tyrosine receptor kinase; ORR: overall response rate; OS: overall survival; PDGFR- $\beta$ : platelet-derived growth factor receptor $\beta$; PDL1: programmed cell death 1 ligand; PET: positron emission tomography; mPFS: median progression free survival; RT: radiotherapy; TIE2: tyrosine-protein kinase receptor Tie-2; VEGF: vascular endothelial growth factor

\section{Background}

Cholangiocarcinoma is a malignancy that affects the hepatic biliary tract and is known to be a rare, aggressive disease, arising from the intra (ICC) or extrahepatic bile duct. Most patients usually are asymptomatic, and diagnosis occurs when the tumor is already in advanced stage [1]. Although cholangiocarcinoma accounts for only $3 \%$ of all gastrointestinal (GI) cancers, approximately $20 \%$ of GI deaths are due to hepatobiliary malignancies. Recently, an epidemiological analysis showed that cholangiocarcinoma incidence increased between 2001 and 2015, probably related to a better diagnostic arsenal, while these tumors were previously classified as cancers of unknown primary. Multiple risk factors can be implicated in the development of cholangiocarcinoma, such as gallstone disease, primary sclerosing cholangitis, bile duct infections, hepatitis, alcohol abuse, tobacco, and inflammatory bowel disease [2].

Treatment for advanced cholangiocarcinoma is limited. Cisplatin and gemcitabine (CG) is indicated for first line therapy (overall survival [OS] of 11.7 months) [3], while the combination of Fluorouracil, Leucovorin and Oxaliplatin (mFOLFOX) has been recently 
defined as a second line treatment choice (survival rate of 6 months reaching 50.6\% months) [4]. However, no other systemic therapy has been supported by robust data, motivating the research for new treatment options, especially in the field of molecular alterations that drive tumor development and growth. In this context, vascular endothelial growth factor (VEGF) overexpression has been found to be present in over $50 \%$ of cholangiocarcinoma and treatment with regorafenib, an anti-VEGF drug, demonstrated only 15.6 weeks of OS in patients who had failed at least 1 previous systemic chemotherapy (CT) treatment [5]. In this case report, we discuss an unexpected disease control and treatment response in a patient with advanced ICC that started regorafenib as a third line therapy and remains with stable disease for more than 16 months.

\section{Case Presentation}

A 49-year-old woman who had a cholecystectomy secondary to gallstones in October 2011 was diagnosed 2 years later with ICC. The patient was asymptomatic, and diagnosis was made due to an imaging finding. A $5.0 \mathrm{~cm}$ lesion located in the hepatic segment $\mathrm{IVb} / \mathrm{V}$ was resected and also regional lymphadenectomy was performed, in which 2 compromised lymph nodes were identified. Pathological staging was pT2pN1. She started radiotherapy (RT) and adjuvant chemotherapy with capecitabine. After 9 months of follow up, the patient had a unique lesion progression in liver. First line treatment with CG was prescribed, which resulted in hepatic lesion reduction, allowing the patient to initiate radiofrequency ablation after CT completion.

The patient had been on exclusive clinical follow up for 2 years, when new lesions appeared in liver, bones and lymph nodes, characterizing a low volume progressive disease. She was reexposed to CG in an attempt to achieve the same previous clinical benefit, however only partial response was observed in positron emission tomography (PET) scan, showing metastatic involvement in L4 with increased risk for pathological fracture, hepatic lesion reduction and disappearance of fluorodeoxyglucose (FDG) uptake in the abdominal lymph nodes. The patient received zoledronic acid to prevent skeletal-related events and palliative RT to treat bone metastasis. Moreover, myelotoxicity and nausea induced by CT were limiting.

Paraffin-embedded tumor blocks were obtained and sent to a comprehensive genomic profiling test that uses next generation sequencing (NGS) technology.

Results reported IDH1, FOXP1 and TP53 mutations, but no therapy with potential clinical benefit was identified. This test also showed stable microsatellite status and low tumor mutation burden. Immunohistochemistry analysis for programmed cell death 1 ligand (PD-L1) expression (SP263 assay) was also negative. Hence, a combination of leucovorin, 5-fluorouracil and irinotecan (FOLFIRI) was started, but the disease progressed after 7 cycles. Considering a satisfactory performance status (ECOG 1) maintained during this period, the patient started regorafenib $120 \mathrm{mg}$ orally per day. After 3 months of treatment, PET scan showed objective metabolic response in all secondary lesions. The patient has been receiving regorafenib for 16 months and remains with stable disease and excellent tolerance to treatment.

\section{Discussion}

Cholangiocarcinoma is a rare disease, and, unfortunately, there is a paucity of treatments available for advanced unresectable or metastatic disease [6]. The median overall survival with the standard of care treatment for this group of patients is less than one year [3]. Data from 981 patients diagnosed with stage IV ICC between 2010 and 2015 showed that most frequent site of metastasis were liver (57.9\%), followed by lung (44.5\%) and bones (29.7\%). Brain was a rare site of metastasis (1.6\%). In this analysis, the median survival was 6 months (95\% CI: 5.434-6.560). The overall survival by site of metastasis differed: 6 months for liver, 6 months for lung, and 4 months for bone metastasis $(\mathrm{p}=0.011)$ [7].

The combination of CG is the standard of care first-line treatment for locally advanced or metastatic cholangiocarcinoma. The use of this regimen showed an improvement of 3.6 months in median overall survival versus placebo (11.7 vs 8.1 months; HR 0.46 ; $95 \%$ IC $0.52-0.80 ; \mathrm{p}<0.001$ ). There was also a higher tumor control rate among patients treated with this chemotherapy (81.4\% vs 71.8\%, p=0.049) [3]. However, the second line treatment has not been supported by robust data, until recently [8]. The ABC 06 trial had established a second line treatment with improvement in overall survival after treatment with CG. This study randomized patients with metastatic biliary tract cancers for active symptom control (ASC) or ASC associated with mFOLFOX. The 6 months and 12 months survival rate were $50.6 \%$ and $25.9 \%$ in the mFOLFOX arm, while in the ASC arm these rates were $35.5 \%$ and $11.4 \%$, respectively [4].

The interest for molecular alterations that drive tumor development and growth is progressively increasing across all the types of tumors. In cholangiocarcinoma, this field of knowledge is also enhancing. An analysis of a 410 cancer-associated genes next-generation sequencing assay was performed from 195 patients, the majority of them had ICC (81\%). Patterns of genetic alterations were differed between intrahepatic and extrahepatic cholangiocarcinoma. In extrahepatic tumors, the most common alterations were in KRAS, SMAD4 and STK11. In ICC, the most frequently altered genes were IDH1 (30\%), ARID1A (23\%) BAP1 (20\%), TP53 (20\%) and FGFR2 gene fusions (14\%). OS was not different for patients who harbored IDH1, FGFR2, BAP1, ATM, ARID1A and TP53 alterations. However, OS was significantly shorter for those who harbored alterations in CDKN2A/B, ERBB2 and KRAS. Potentially targetable genes were found in $47 \%$ of patients. Clinical trial enrollment or biomarker directed therapy was possible in $16 \%$ of them [9]. 
In addition, VEGF overexpression has been found to be present in over $50 \%$ of cholangiocarcinoma [10]. Regorafenib is an oral multi-kinase drug that potentially inhibits VEGF receptors (1-3, tyrosine-protein kinase receptor Tie-2 [TIE2]), stromal factors (platelet-derived growth factor receptor $\beta$ [PDGFR- $\beta$ ] and fibroblast growth factor receptor 1 [FGFR1]), and multiple oncogenic kinases. Based on this data, regorafenib was tested in a single-arm, phase II trial in 43 patients with advanced/metastatic biliary tract cancer who failed at least 1 previous systemic chemotherapy treatment. Twenty-seven patients had primary ICC. The overall response rate (ORR) in this study was $11 \%$ and $44 \%$ of the patients had stable disease. The median progression free survival (mPFS) was 15.6 weeks (IC 90\% 12.9-24.7 weeks) and the median OS was 15.6 weeks (IC $90 \% 23.3-74.3$ weeks). In 18 months, $32 \%$ of the patients were alive [5]. Our patient has been benefiting greatly from this treatment, achieving survival over the expected.

One hypothesis about this increased response and improved survival with regorafenib is related to the tumor suppressor gene p53 functional loss, identified in the NGS test. Although no therapies are approved to address TP53 mutation or loss, tumors with this alteration can upregulate VEGF-A and VEGFR2, which may be a target to anti-VEGF drugs. A prospective study enrolled 500 patients to receive targeted agents based on the results from a 236 genes next-generation sequencing test. Treatment occurred in 188 patients, in which 106 had tumors harboring TP53 mutations. Outcome parameters analyzed were rate of stable disease $\geq 6$ months/partial response/complete response; length of time to failure; overall survival. Use of VEGF/VEGFR inhibitors was independently associated with improvement in all these parameters. However, not statistically outcome improvement was observed in patients with TP53 wild-type tumors. The authors concluded that TP53 mutations may predict sensitivity to VEGF/VEGFR inhibitors [11], which could be a hypothesis to the unexpected response demonstrated by this case report.

Another target that has been studied with successful results is FGFR2. A phase II trial with an FGFR inhibitor, BGJ398, which included 61 patients with advanced cholangiocarcinoma that harbored FGFR2 fusions, mutations or amplifications, showed ORR of $14.8 \%$ and disease control rate of $75.4 \%$. Estimated median PFS was 5.8 months [12]. Another phase II trial, FIGHT-2, enrolled 146 patients in three different cohorts: cohort A (FGFR2 gene rearrangements/fusions), B (other FGF/FGFR gene alterations), or C (no FGF/FGFR gene alterations). All patients were exposed to pemigatinib, an oral FGFR 1,2 and 3 inhibitor. In cohorts $B$ and C, no patients achieved response. Nevertheless, in cohort A, ORR was $35.5 \%$, with disease control rate of $82 \%$. Besides, the mPFS and mOS in this cohort were 6.9 months and 21.1 months, respectively (OS does not mature at cutoff). These data support the use of an FGFR2 inhibitor for previously treated patients with advanced cholangiocarcinoma harboring FGFR2 gene rearrangements/ fusions [13].
Isocitrate dehydrogenase (IDH) alterations have also been studied as potential targets in ICC. The multi tyrosine kinase dasatinib was shown to be active in tumors with IDH mutations [14]. Another effective drug in this scenario is ivosidenib, a firstin-class oral inhibitor of the mutant IDH1 (mIDH1) protein. A phase III, placebo-controlled, randomized clinical trial randomized 185 patients with advanced cholangiocarcinoma with mIDH1 to ivosidenib or placebo. The majority of the patients had ICC (91\%) and $42 \%$ were treated with 2 previous therapies. PFS rates at 6 and 12 months were $32 \%$ and $21,9 \%$ with ivosidenib, while in placebo there were no patient's progression-free for greater than 6 months (HR: $0.37 ; 95 \%$ CI $0.25,0.54 ; \mathrm{p}<0.001$ ). There was also a favorable trend in OS towards ivosidenib (HR 0.69; p 0.06). There was a $57 \%$ crossover rate from patients in placebo to ivosidenib. This was the first study demonstrating clinical benefit in IDH1 inhibition in this subgroup of patients [15].

Currently, immunotherapy has been widely studied. Biliary tract tumors can be a target to checkpoint inhibitors due to the chronic inflammation involved [16]. Interim results of KEYNOTE-028, a phase $1 \mathrm{~b}$ trial accessing the use of pembrolizumab across PD-L1 positive tumors, showed an ORR of $17 \%$. The population exposed to pembrolizumab was heavily pretreated, with $38 \%$ of patients with more than 3 previous therapies [17]. For tumors with DNA mismatch repair deficiency (dMMR) or high microsatellite instability (MSI-H), the therapeutic indication for pembrolizumab is based on the agnostic approval (Figure 1). MSI-H occurs in approximately $3 \%$ of the biliary tract cancers [18]. There are several other potential targets which are currently being studied for advanced cholangiocarcinoma, such as neurotrophic tyrosine receptor kinase (NTRK) fusions [19], MET, PI3K, BRCA and BAP1 $[18,20]$.

\section{Conclusion}

Our patient with advanced ICC that was refractory to 2 lines of treatment, demonstrated prolonged survival with regorafenib in a third line setting. Therapeutic scenario of cholangiocarcinoma has been changing. Ongoing clinical trials results are awaited in an attempt to define which molecular alterations are important to plan better treatments, selecting patients for targeted therapies, immunotherapy, or even its combination with CT. Currently, in the third line treatment, next-generation sequencing assays, mismatch repair protein expression and immunohistochemistry for PDL1 should be considered, in order to personalize and optimize oncologic therapy.

\section{Authors' Contributions}

Costa CM and Gongora ABL were responsible for the data collection and writing. Lopes $\mathrm{CDH}$ was responsible for writing assistance. Salgues ACR analyzed the manuscript. Capareli FC is the patient's personal oncologist and also analyzed the references collected, the data selected and the manuscript. All authors read and approved the final manuscript. 

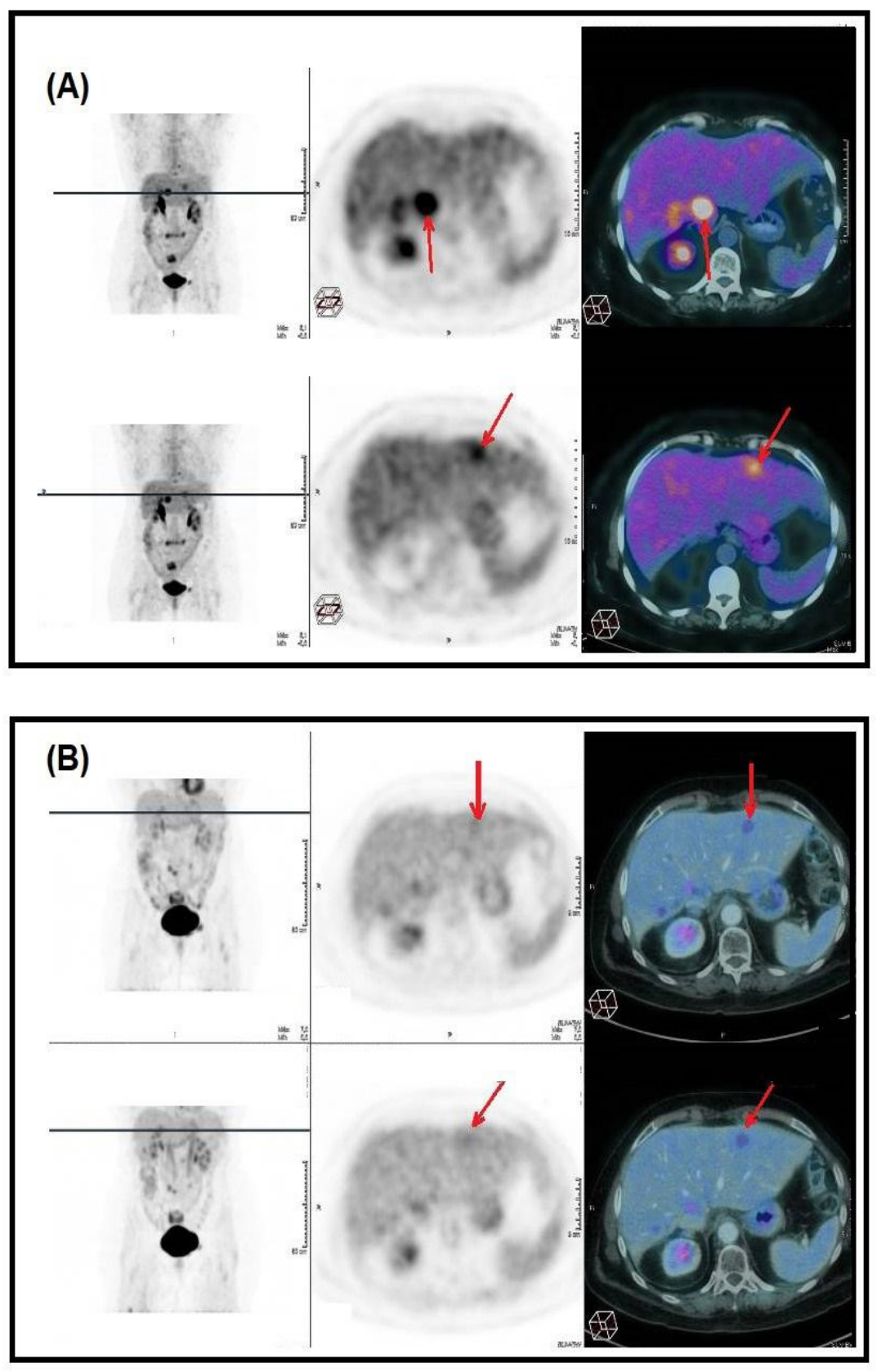

Figure 1: (A) PET scan after 7 cycles of FOLFIRI showing higher FDG uptake in 2 hepatic nodules. (B) Image control after 16 months of regorafenib treatment indicating metabolic response and stable morphological lesions.

\section{References}

1. Niederhuber J, Armitage J, Doroshow J, Kastan M, Abeloff's TJ (2020) Clinical Oncology. In: (6 ${ }^{\text {th }}$ edn), Liver and Bile Duct Cancer, Elsevier, pp. 1314-1341.

2. Patel N, Benipal B (2019) Incidence of cholangiocarcinoma in the USA from 2001 to 2015: A US cancer statistics analysis of 50 states. Cureus 11(1): e3962.

3. Valle J, Wasan H, Palmer DH, Cunningham D, Anthoney A, et al. (2010) Cisplatin plus gemcitabine versus gemcitabine for biliary tract cancer. $\mathrm{N}$ Engl J Med 362(14): 1273-1281.
4. Lamarca A, Palmer DH, Wasan HS, Ross PJ, Ma YT, et al. (2019) ABC-06|A randomised phase III, multi-centre, open-label study of active symptom control (ASC) alone or ASC with oxaliplatin/5-FU chemotherapy (ASC+mFOLFOX) for patients (pts) with locally advanced/metastatic biliary tract cancers (ABC) previously-treated with cisplatin/ gemcitabine (CisGem) chemotherapy. JCO 37(15_suppl): 4003.

5. Sun W, Patel A, Normolle D, Patel K, Ohr J, et al. (2019) A phase 2 trial of regorafenib as a single agent in patients with chemotherapy-refractory, advanced, and metastatic biliary tract adenocarcinoma. Cancer 125(6): 902-909. 
6. Rizvi S, Khan SA, Hallemeier CL, Kelley RK, Gores GJ (2018) Cholangiocarcinoma-evolving concepts and therapeutic strategies. Nat Rev Clin Oncol 15(2): 95-111.

7. Yan X, Wang P, Zhu Z, Ning Z, Xu L, et al. (2019) Site-specific metastases of intrahepatic cholangiocarcinoma and its impact on survival: a population-based study. Future Oncology 15(18): 2125-2137.

8. Ghidini M, Pizzo C, Botticelli A, Hahne JC, Passalacqua R, et al. (2018) Biliary tract cancer: current challenges and future prospects. Cancer Manag Res 11: 379-388.

9. Lowery MA, Ptashkin R, Jordan E, Berger MF, Zehir A, et al. (2018) Comprehensive molecular profiling of intrahepatic and extrahepatic cholangiocarcinoma: Potential targets for intervention. Clin Cancer Res 24(17): 4154-4161.

10. Yoshikawa D, Ojima H, Iwasaki M, Hiraoka N, Kosuge T, et al. (2008) Clinicopathological and prognostic significance of EGFR, VEGF, and HER2 expression in cholangiocarcinoma. Br J Cancer 98(2): 418-425.

11. Wheler JJ, Janku F, Naing A, Li Y, Stephen B, et al. (2016) TP53 alterations correlate with response to VEGF/VEGFR inhibitors: Implications for targeted therapeutics. Mol Cancer Ther 15(10): 2475-2485.

12. Javle M, Lowery M, Shroff RT, Weiss KH, Springfeld C, et al. (2018) Phase II Study of BGJ398 in Patients With FGFR-Altered Advanced Cholangiocarcinoma. J Clin Oncol 36(3): 276-282.

13. Vogel A (2019) FIGHT-202: a phase 2 study of pemigatinib in patients (pts) with previously treated locally advanced or metastatic cholangiocarcinoma (CCA). Barcelona, Spain.
14. Saha SK, Gordan JD, Kleinstiver BP, Vu P, Najem MS, et al. (2016) Isocitrate dehydrogenase mutations confer dasatinib hypersensitivity and SRC dependence in intrahepatic cholangiocarcinoma. Cancer Discov 6(7): 727-739.

15. Abou-Alfa G (2019) ClarIDHy: A global, phase 3, randomized, doubleblind study of ivosidenib (IVO) vs placebo in patients with advanced cholangiocarcinoma (CC) with an isocitrate dehydrogenase 1 (IDH1) mutation. Abstract LBA10_P presented at: 2019 ESMO Congress, Barcelona, Spain.

16. Goldstein D, Lemech C, Valle J (2017) New molecular and immunotherapeutic approaches in biliary cancer. ESMO Open 2(Suppl 1): e000152.

17. Bang YJ, Doi T, Braud FD, Piha-Paul S, Hollebecque A, et al. (2015) 525 Safety and efficacy of pembrolizumab (MK-3475) in patients (pts) with advanced biliary tract cancer: Interim results of KEYNOTE-028. European Journal of Cancer 51: S112.

18. Adeva J, Sangro B, Salati M, Edeline J, La Casta A, et al. (2019) Medical treatment for cholangiocarcinoma. Liver Int 39(S1): 123-142.

19. Amatu A, Sartore-Bianchi A, Siena S (2016) NTRK gene fusions as novel targets of cancer therapy across multiple tumour types. ESMO Open 1(2): e000023.

20. DeLeon TT, Ahn DH, Bogenberger JM, Anastasiadis PZ, Arora M, et al. (2018) Novel targeted therapy strategies for biliary tract cancers and hepatocellular carcinoma. Future Oncology 14(6): 553-566.

For possible submissions Click below:

Submit Article 